\title{
Modernes CML-Management
}

\section{Früheres und tieferes Ansprechen unter Dasatinib - Evidenz für den langfristigen Therapieerfolg}

Die DGHO-Jahrestagung fand vom 19.-23. Oktober in Stuttgart statt und bot rund 4500 Experten die Gelegenheit, aktuelle Studiendaten und zukunftsweisende Behandlungskonzepte in der Hämatoonkologie zu diskutieren. Beim Therapiemanagement der chronischen myeloischen Leukämie (CML) zeichnet sich derzeit ein Paradigmenwechsel ab, der sowohl die Definition des «Standard of Care» für neu diagnostizierte Patienten als auch die Frage betrifft, wann Patienten mit unzureichendem Imatinib-Ansprechen auf einen modernen, hochpotenten Tyrosinkinaseinhibitor wie Dasatinib (Sprycel ${ }^{\circledR}$ ) umgestellt werden sollten [1].

Die Entschlüsselung der Bedeutung des Onkogens BCR-ABL für die CML-Pathogenese der meisten Patienten und die Ableitung eines Therapieprinzips, das sich gegen das chimäre Proteinprodukt dieses Fusionsgens - eine Tyrosinkinase - richtet, führte zu einer erheblichen Verbesserung der Lebenserwartung [2]. Mit der Einführung der Tyrosinkinaseinhibitoren (TKIs) in der chronischen Phase der Erkrankung hat die CML die Wandlung von einer neoplastischen Erkrankung, die unbehandelt unweigerlich zum Tode führte, zur derzeit prognostisch günstigsten Leukämieform überhaupt vollzogen [3].

Allerdings stellte sich heraus, dass die Patienten kein homogenes Ansprechverhalten auf den Erstgenerations-TKI Imatinib zeigen: Ein signifikanter Anteil der CML-Patienten zeigt ein nur suboptimales Ansprechen [4], lediglich eine Minderheit erreicht das

Molekulares Ansprechen nach 3 Monaten*

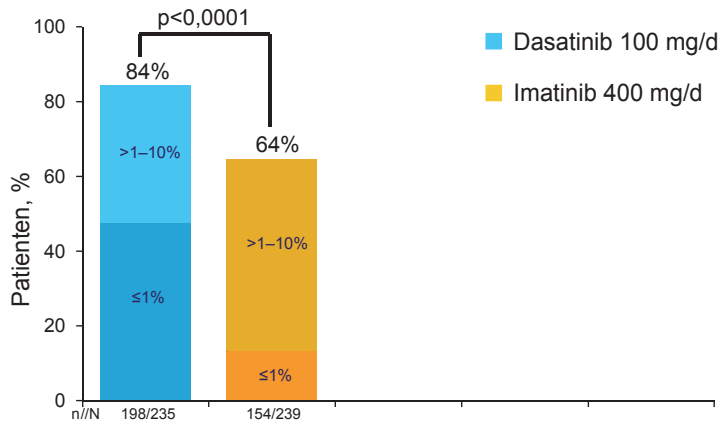

$\leq 10 \%$ BCR-ABL nach 3 Monaten

* Kalkulation basiert auf Gesamtzahl der evaluierten Patienten mit PCR-Analyse nach 3 Monaten

Abb. 1. DASISION-Studie: Molekulares Ansprechen gegenüber Dasatinib (Sprycel ${ }^{\circledR}$ ) nach 3 Monaten, nach [11]
Kriterium des kompletten molekularen Ansprechens (CMR) [5]. Zudem wurde beobachtet, dass das Imatinib-Ansprechen für einige Patienten nicht von Dauer ist [6]. Überdies können Primärresistenzen bzw. sekundäre Resistenzentwicklungen oder Unverträglichkeiten mit der Medikation das Therapie-Outcome belasten [7].

Inzwischen wurden potentere TKIs der 2. Generation entwickelt, die über eine verbesserte Wirksamkeit verfügen und zu einer intensivierten Suche nach prognostischen Markern für suboptimales Therapieansprechen geführt haben [8]. Denn wie sich in Verlaufsanalysen zeigte, war ein suboptimales Imatinib-Ansprechen oder -Therapieversagen nach 3, 6, 12 oder 18 Monaten mit deutlichen Nachteilen für das progressionsfreie sowie Gesamtüberleben vergesellschaftet [9].

\section{BCR-ABL nach 3 Monaten als frühzeitiges Prognosekriterium}

Zur Differenzierung zwischen Patienten mit suboptimalem oder optimalem Ansprechen sowie Therapieversagen können hämatologische, zytogenetische und molekulare Parameter zu definierten Zeitpunkten herangezogen werden [1]. Derzeit wächst die Evidenz, dass die Tiefe der erreichten Remission und der möglichst frühe Zeitpunkt für die tiefe Remission für die Langzeitprognose des CMLPatienten weichenstellend sind. Die Deutsche CML-Studiengruppe hat kürzlich die frühe prognostische Relevanz des molekularen Ansprechens gegenüber Imatinib, wie sie sich mithilfe des BCR-ABLTranskriptionslevels nach der internationalen Skala (BCR-ABLIS) bestimmen lässt, herausgearbeitet: Demnach profitierten Patienten, die im Rahmen der CML-IV-Studie nach 12-monatiger Therapie einen BCR-ABL-Wert von $\leq 1 \%$ erreichen, von einem höheren 3-Jahres-Überleben als Patienten, die zu diesem Zeitpunkt nur einen BCR-ABL-Wert von $>1 \%$ erreicht hatten [10].

Die jüngsten Verlaufsanalysen der CML-IV-Studie ergaben schließlich, dass bereits der BCR-ABL-
Wert nach 3 Monaten die Charakterisierung einer «Hochrisiko-Gruppe» für das therapeutische Ansprechen zulässt und sich die « $\leq 1 \%$-Gruppe» und die «1-10\%-Gruppe» beim Gesamtüberleben nicht signifikant unterscheiden [1]: So riskierten diejenigen $\mathrm{Pa}-$ tienten, die den kritischen Schwellenwert von $<10 \%$-BCR-ABL zum 3-Monatszeitpunkt nicht erreichten, ein signifikant schlechteres 5-Jahres-Gesamtüberleben gegenüber Patienten, die den PCR-Meilenstein $\mathrm{zu}$ diesem Zeitpunkt erreicht hatten $(\mathrm{p}=0,012)$.

Vor diesem Hintergrund ziehen die Studienautoren die Schlussfolgerung, dass die Gruppe der «Hochrisiko-Patienten» schon zu diesem Zeitpunkt im Zuge einer «Hit-hard-andearly»-Rationale von der Umstellung auf effektivere Therapieoptionen profitieren könnte [1].

\section{Schnelles und tiefes Ansprechen für bessere Langzeitprognose}

Die Korrelation zwischen dem BCR-ABLTiter nach 3 Monaten und dem langfristigen Therapieergebnis wurde jetzt auch durch eine bei der diesjährigen ASCO-Jahrestagung vorgestellte Follow-up-Analyse der DASISION*-Studie bestätigt [11]: Unter Dasatinib wiesen $20 \%$ mehr CML-Patienten einen BCR-ABL-Wert von $\leq 10 \%$ auf als unter Imatinib nach 3 Monaten (84\% vs. $64 \% ; p=0,0001 ;$ Abb. 1). Und tatsächlich korrelierte das tiefe molekulare Ansprechen zum kritischen 3-Monatszeitpunkt signifikant mit dem Langzeit-Outcome nach 3 Jahren - sowohl im Hinblick auf das progressionsfreie als auch auf das Gesamtüberleben (Abb. $2 ; p=0,0003$ für beide). Die Followup-Langzeitanalyse wird auch weiterhin fortgesetzt [11]

In der randomisierten, offenen Phase-III-Studie DASISION war Dasatinib $100 \mathrm{mg} / \mathrm{d}$ mit Imatinib $400 \mathrm{mg} / \mathrm{d}$ bei insgesamt 519 Patienten mit neu diagnostizierter Ph+ CML in der chronischen Phase verglichen worden: Die 1-Jahres-Daten hatten bereits zur Erstlinien-Zulassung von Dasatinib geführt [12]. Die neu diagnostizierten Patienten hatten unter der Behandlung mit Dasatinib nach 12 Monaten signifikant häufiger ein komplettes zytogenetisches Ansprechen erreicht (CCyR,

Dasatinib versus Imatinib Study in Treatment-Naïve CML-Patients 

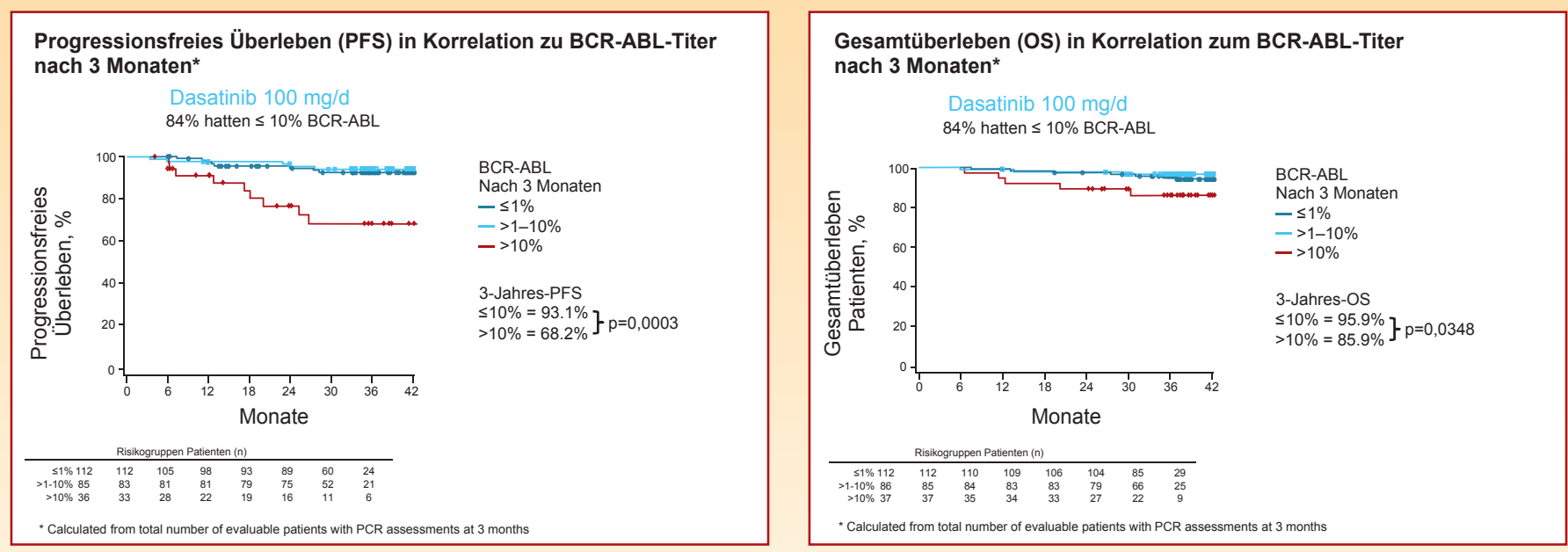

Abb. 2. Frühes molekulares Ansprechen auch in der First-Line-Therapie mit Dasatinib prädiktiv für das progressionfreie und Gesamtüberleben der Patienten: PFS und OS in Korrelation zum 3-Monats-Ansprechen, nach [11].

complete cytogenetic response) als unter Imatinib ( $77 \%$ vs. $66 \%$; $p=0,007)$. Überdies hatten Dasatinib-behandelte Patienten seltener eine Transformation in die CML-Akzeleration oder Blastenkrise. Dabei korrelierten niedrige Transformationsraten mit dem zuvor tieferen Therapieansprechen nach 3 Monaten.

\section{Stark vorbehandelte Patienten profitieren ebenfalls}

Neben der First-Line-Therapie bietet Dasatinib auch eine wichtige Therapieoption für vorbehandelte Patienten mit Ph+ CML in der chronischen Phase und Therapieresistenz, Unverträglichkeit oder suboptimalem Ansprechen gegenüber Imatinib. Mittlerweile umfasst die Langzeiterfahrung mit Dasatinib in der CMLZweitlinientherapie 6 Jahre und bietet somit das längste Follow-up überhaupt, das derzeit zu einem TKI der 2. Generation bei Patienten mit Imatinib-Resistenz oder Imatinib-Unverträglichkeit verfügbar ist. Dabei erreichten selbst stark vorbehandelte Patienten unter der Second-Line-Therapie mit Dasatinib die hohe Gesamtüberlebensrate von 71\% [13].

Eine Analyse der BCR-ABL-Werte hat auch hier zeigen können, dass ein früheres und tieferes Ansprechen mit besseren Langzeitergebnissen korreliert: Patienten, die schon bei $\leq 3$ Monaten einen BCR-ABL $\leq 10 \%$ aufwiesen, profitierten sowohl von einem verlängerten progressionsfreien Überleben als auch einem verlängertem Gesamtüberleben ( $p<0,001$ für beide). Das günstige Sicherheits- und Verträglichkeitsprofil von Dasatinib blieb auch nach 6 Jahren stabil und zeigte keine wesentlichen Abweichungen von den bisherigen Daten [13]. Und: Sowohl im First-Line- als auch im Second-Line-Setting war die Behandlung mit Dasatinib mit nur niedrigen $\mathrm{Ab}$ bruchraten aufgrund von Nebenwirkungen assoziiert $[12,14]$.

\section{Adhärenz im \\ CML-Langzeitmanagement}

Die orale Verfügbarkeit der TKIs ermöglicht dem Patienten eine in hohem Maß eigenverantwortliche Therapie. Gleichzeitig hängt der Therapieerfolg von der regelmäßigen Einnahme der Medikation sowie dem darunter konstanten Wirkspiegel ab und damit in hohem $\mathrm{Ma} 3$ von der therapeutischen Adhärenz des Patienten - einer Herausforderung, die bereits von anderen chronisch-internistischen Erkrankungen bekannt ist, die ebenfalls eine Langzeitmedikation erforderlich machen [7].

So hat eine britische Studie des HammersmithHospitals in London aufgezeigt, dass schon eine relativ kleine Abweichung vom verordneten Therapieregime - in diesem Fall eine Adhärenz von $\leq 90 \%$ - den klinischen Nutzen der Therapie in Frage stellen kann [15]. Auch nahm die Wahrscheinlichkeit, eine erreichte CCyR wieder zu verlieren, bei einer Adhärenz von weniger als $85 \% \mathrm{zu}$. Die Beweggründe für non-adhärentes Verhalten sind vielschichtig und häufig sehr individuell: Sie können von therapiebezogenen Ursachen wie Unverträglichkeitsproblemen mit der Medikation über patientenbezogene Faktoren bis hin zu sozioökonomischen und psychosozialen Faktoren reichen [16]. Trotz der insgesamt guten Verträglichkeit von TKIs können auch weniger stark ausgeprägte Nebenwirkungen, die aber persistieren, den Patienten in seiner Lebensqualität beeinträchtigen und die Adhärenz ungünstig beeinflussen [17]. Zudem sind bei der Therapiewahl die unterschiedlichen Nebenwirkungsprofile der TKIs in Abhängigkeit von den individuellen internistischen Begleiterkrankungen der Patienten zu berücksichtigen [7]. Einen wichtigen Beitrag zur Adhärenz-Verbesserung können zum Beispiel psychoedukative Maßnahmen leisten, indem sie den Patienten über das therapeutische Benefit der Langzeit- medikation und potenzielle Nebenwirkungen sowie ggf. den adäquaten Umgang mit ihnen aufklären [7]. Daneben sollten die Behandler ihre Patienten auch auf psychoonkologische Betreuungsangebote aufmerksam machen, wo Patienten eine individuelle Beratung erfahren können.

\section{Dr. Yuri Sankawa, Stuttgart}

\section{Referenzen}

Hanfstein B et al.: Leukemia 2012;26:1-7.

2 Hehlmann R et al.: Lancet 2007;370:342-50.

3 Hochhaus A.: Hematology Am Soc Hematol Educ Program 2011;2011:128-35.

4 O'Brien SG et al.: N Engl JMed 2003;348:994-1004.

5 Cross NCP et al.: Leukemia 2012;26:2172-5.

6 Hughes TP et al.: Blood 2010;116:3758-65.

Jabbour E et al.: Leukemia 2011;25:201-10.

8 Fabarius A et al: Blood 2011:118:6760-68.

9 Baccarani M et al.: J Clin Oncol 2009;27:6041-51.

10 Hehlmann R et al.: J Clin Oncol 2011;29:1634-42.

11 Hochhaus A et al.: ASCO 2012; abstr 6504

12 Kantarjian HM et al.: Blood 2012;119:1123-9.

13 Shah et al.: 6year follow-up of CA180-034, abstr 6506, ASCO 2012.

14 Shah NP et al.: Haematologica 2010;95:232-40

15 Marin D et al.: Clin Oncol 2010;28:2381-88.

16 WHO: Adherence to long-term therapies.2003.

17 Cornelison M et al.: J Support Oncol 2012;10:14-24.

\section{Impressum}

Modernes CML-Management

Früheres und tieferes Ansprechen unter Dasatinib - Evidenz für den langfristigen Therapieerfolg

PharmaForum in ONKologie 35 | 12 | 12

(C) 2012 by S. Karger Verlag für Medizin und Naturwissenschaften $\mathrm{GmbH}$

Wilhelmstraße 20A

79098 Freiburg, Deutschland

Mit freundlicher Unterstützung durch Bristol-Myers Squibb GmbH \& Co. KGaA.

Verlag, Herausgeber, Redaktion und Verlagsgeschäftsführung übernehmen keine Verantwortung für den Inhalt dieser Rubrik. 